\title{
Prospective study of thyroid functions in multiple sclerosis patients treated with interferon beta in Alexandria University MS clinic
}

\author{
Farouk Talaat and Hany Eldeeb * (D)
}

\begin{abstract}
Background: The incidence of thyroid dysfunction in multiple sclerosis (MS) patients on interferon (as a diseasemodifying line) has been reported in many studies performed on patients already on interferon therapy. Our study adopted a prospective follow-up of the thyroid profile in those patients newly starting interferon beta therapy over 1 year trying to find a correlation between the start of therapy and the occurrence of this dysfunction.

Objectives: To evaluate the incidence of thyroid dysfunction in MS patients before and 1 year after of interferon beta.

Methods: A prospective cohort study on 48 MS patients newly receiving interferon beta therapy through MS clinic were subjected to assessment of thyroid profile including serum TSH, free T3, and T4 at the start of treatment; they were followed-up at 6- and 12-month intervals after the start of therapy.

Results and conclusion: There was a statistically significant increase in serum-free T3 levels in those treated with interferon beta therapy in a follow-up of 1 year. Our work also showed a decrease in serum-free T4 levels without statistical significance. TSH levels showed a tendency towards increase without statistical significance.
\end{abstract}

Keywords: Multiple sclerosis, Thyroid hormones, Interferons beta

\section{Introduction}

Multiple sclerosis (MS) is an inflammatory, neurodegenerative disease characterized by CNS-directed autoimmunity characterized by inflammation, demyelination, and axonal transection. It is characterized pathologically by dendritic cell migration across the blood-brain barrier and induces degeneration of memory $\mathrm{T}$ cells into pro- inflammatory $\mathrm{T}$ helper 1 lymphocytes [1].

Pro-inflammatory cytokines are also involved in the demyelination and axonal loss in MS. A number of disease-modifying therapies have been approved for the

\footnotetext{
* Correspondence: hanys5252@gmail.com

Neurology Department, Faculty of Medicine, Alexandria University, Alexandria, Egypt
}

\section{Springer Open}

treatment of relapsing MS with no significant role in progressive subtypes (either primary or secondary) [2].

Interferon beta (IFN- $\beta$ ) is considered a significant disease-modifying line in treating remitting-relapsing MS, as it maintains the anti-inflammatory status of the immune system by interacting with the IFN $\alpha / \beta$ cell surface receptors. INF B was a matter of research for long time as having a strong correlation with many autoimmune disorders that have been reported in MS patients [3].

Interferon beta (IFN- $\beta$ ) is recommended as a first-line disease-modifying treatment (DMT) for relapsingremitting multiple sclerosis (RRMS) in over 90 countries as it is more convenient economically than other lines and easily adopted by most insurance systems in these countries [4]. It is the disease-modifying line of choice in 
more than 50\% of MS patients in Egypt according to an Egyptian registry published in 2017 [5].

The most frequently reported adverse effects associated with IFN- $\beta$ treatment worldwide are flu-like symptoms, injection site reactions, fatigue, and transient laboratory abnormalities [6].

Autoimmune thyroid diseases (AITDs) in patients with MS as compared with healthy controls has been reported in many old studies that show an increased prevalence of AITD in patients with MS. This concept started to change recently with the appearance of new studies reporting that the rates of AITDs in MS patients are consistent with its prevalence in the general population [7].

IFN- $\beta$ therapy can affect the thyroid function either through its direct thyrotoxic properties or indirectly by inducing autoimmune-mediated thyroid dysfunction. This can be presented as autoimmune thyroiditis either clinically overt manifestations or subclinical laboratory findings [8].

Many studies have found a significantly abnormal thyroid profile in MS patients on interferon beta therapy. There was no clear evidence that abnormal thyroid profile could occur in newly diagnosed cases as a de novo finding attributed only to interferon therapy or it is just an incidental finding. Our study aimed to evaluate, in a prospective pattern, the incidence of thyroid dysfunction, specifically serum levels of thyroid-stimulating hormone (TSH), free thyroxine, and triiodothyronine in MS patients before the start of treatment and track these cases for 1 year to check the occurrence of abnormal thyroid profiles during therapy.

\section{Methods}

The study is a prospective cohort study that was conducted on 48 patients with age ranging from 19 to 48

Table 1 Distribution of the studied cases according to demographic data at initial visit $(n=48)$

\begin{tabular}{ll}
\hline & No. (\%) \\
\hline Gender & $11(22.9)$ \\
Male & $37(77.1)$ \\
Female & \\
Age (years) & $30.5(19-48)$ \\
Median (min.-max.) & $31.2 \pm 6.6$ \\
Mean \pm SD & \\
Age of onset & $26.5(17-42)$ \\
Median (min.-max.) & $27 \pm 6.4$ \\
Mean \pm SD & \\
Family history & $41(85.4)$ \\
Negative & $7(14.6)$ \\
\hline
\end{tabular}

years old, recruited from our MS clinic during a period of 6 month starting from June 2017 till December 2017 and they were followed-up for 1 year after recruitment which ended at December 2018. The patients were drug naïve as regards disease-modifying therapies. They were collected randomly of both sex diagnosed with multiple sclerosis according to Macdonald's criteria 2010 [9].

All patients were subjected to full medical history taking, thorough clinical neurological examination, expanded disability status scale (EDSS) at the initial assessment visit [10].

Laboratory investigations and radiological investigations as MRI brain and cervical spine were done at the first clinical interview using magnetic resonance imaging with diffusion-weighted image [using a Philips Achieva 1.5 T MRI scanner (Philips Healthcare, Netherland)] were done in the initial assessment visit.

For laboratory work, $4 \mathrm{ml}$ of venous blood samples were collected at room temperature by venipuncture for measurement of free T3, T4, and TSH. Samples were sent to the clinical pathology lab of our university hospital. Serum-free T4, free T3, and TSH were measured. The kit used was a double-antibody sandwich enzymelinked immunosorbent assay (ELISA) to assay level of human free T3, free T4, and TSH in samples.

Follow-up assessments of this thyroid profile were done at 6- and 12-month intervals after the start of therapy. Anti-thyroglobulin antibodies were not sampled as the investigated patients did not show evidence of thyroid dysfunction during follow-up assessments which was defined as evident increase in serum levels of TSH more than $2 \mathrm{mU} / \mathrm{L}$.

Medical informed consents were taken from all patients before the procedures. Ethical approval was obtained from our faculty ethical committee. Exclusions criteria were history of any thyroid diseases (autoimmune thyroid diseases, thyroid tumors, and thyroiditis), family history of thyroid diseases, or any medical procedures affecting thyroid profile like previous thyroid operation or radioactive iodine, history of exposure to radiations or chemotherapy, drugs affecting thyroid

Table 2 Distribution of the studied cases according to number of relapses and EDSS at initial visit $(n=48)$

\begin{tabular}{|c|c|}
\hline & No. (\%) \\
\hline \multicolumn{2}{|l|}{ Total no of relapses } \\
\hline 1 & $4(8.3)$ \\
\hline $1+O C B$ & $3(6.3)$ \\
\hline$>1$ & $41(85.4)$ \\
\hline \multicolumn{2}{|l|}{ EDSS } \\
\hline Median (min.-max.) & $1.5(0-6)$ \\
\hline Mean \pm SD & $1.7 \pm 1.2$ \\
\hline
\end{tabular}


function, history of other autoimmune disorder as systemic lupus erythromatosis, rheumatoid arthritis, other neurological concomitant disease, history of interferon therapy, pregnancy, and EDSS $>6$.

\section{Statistical analysis}

Data were fed to the computer and analyzed using IBM SPSS software package version 20.0. (Armonk, NY; IBM Corp,) Qualitative data were described using number and percent. Quantitative data were described using range (minimum and maximum), mean, standard deviation, and median. Significance of the obtained results was judged at the $5 \%$ level. Friedman test for abnormally distributed quantitative variables, to compare between more than two periods or stages and post hoc test (Dunn's) for pairwise comparisons.

\section{Results}

The study was performed on 48 drug-naïve patients visiting MS clinic from June to December 2017. They included 37 females and 11 males, with age ranging from 19 to 48 years with a mean of $31.6 \pm 6.5$ years. They reported the age of their first symptoms from 17 to 42 years with a mean of $27.3 \pm 6.4$ years. Negative family history was reported in 41 of our patients (Table 1).

At initial assessment visits, all the patients were asked for their total number of previous relapses and oligo-clonal band status of CSF analysis (which is done in our MS clinic in some patients for confirming initial diagnosis of MS). Total number of relapses showed one relapse only in 4 patients, one relapse with positive oligo-clonal bands in CSF in 3 patients, and more than one relapse in the rest of our patients. EDSS score in our patients ranged from 0 to 6 with a median of 1.5 (Table 2).
In correspondence to the type of disease-modifying drug used, our patients were on interferon therapy, 19 of them were on subcutaneous injection with interferon $\beta$ 1a $44 \mu \mathrm{g}$ three times per week (Rebif ${ }^{\circ}$ ), 21 were on subcutaneous injection interferon $\beta$-1b $250 \mu \mathrm{g}$ every other day (Betaferon ${ }^{\circ}$ ), and 8 were on intramuscular interferon $\beta$-1a $30 \mu$ g once per week (Avonex ${ }^{\circ}$ ) (Fig. 1).

Upon assessing thyroid stimulating hormone TSH in the whole studied group, it showed a statistically nonsignificant decrease through the study from a mean of $1.6 \pm 1.6 \mathrm{mu} / \mathrm{L}$ to a mean of $1.4 \pm 1$ and $1.42 \pm 1.07 \mathrm{mu} /$ 1 at 6 and 12 months intervals, respectively ( $p$ value $=$ 0.062) (Table 3, Figs. 2 and 3).

This decrease was also reported when we compared each specific treatment separately at the same intervals. It was statistically significant only in those treated with Rebif after 12 month ( $p$ value $=0.028)$ (Table 3, Figs. 2 and 4).

There was a statistically significant increase in free T3 levels in the studied patients collectively $(p<0.001)$ and it was also noticed in both groups treated with Betaferon $(p=0.010)$ and Rebif $(p=0.030)$. It was also noticed in those treated with Avonex but without statistical significance $(p=0.857)$ (Table 3).

As regards free $\mathrm{T} 4$, there was also a statistically significant increase in its serum level in all patients of the study collectively $(p=0.049)$. Upon comparing it in different lines of treatment, it still showed a tendency towards increase but with no statistical significance. (Table 3, Figs. 2 and 5)

\section{Discussion}

Our study was a prospective cohort study that was conducted on 48 patients with age ranging from 19 to 48 years old, recruited from the MS clinic during a period of 6 months starting from June 2017. They were followed-up at 6- and 12-month intervals from

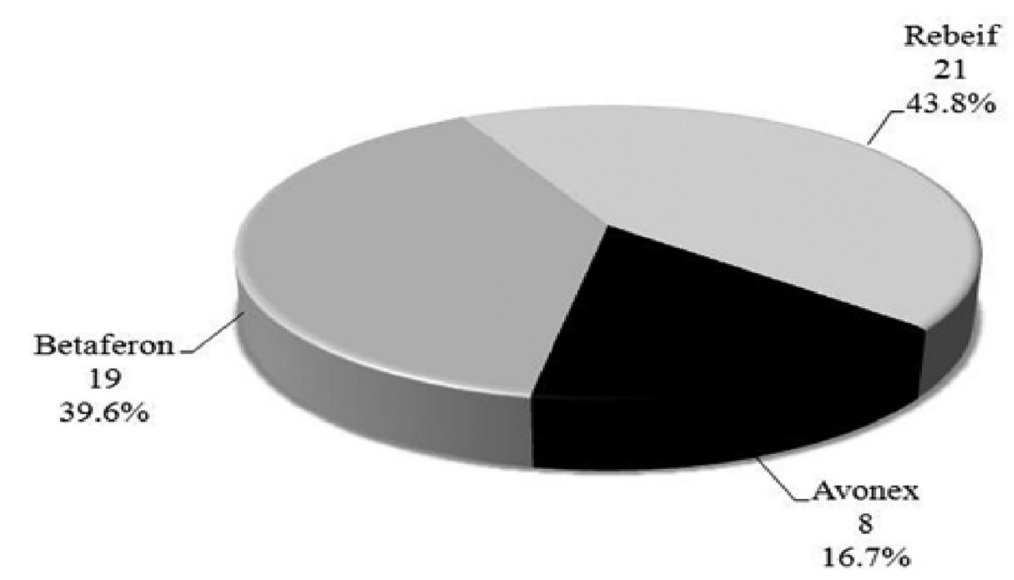

Fig. 1 Distribution of the studied cases according to type of DMT received at the baseline visit $(n=48)$ 
Table 3 Comparison between the different groups at baseline and follow-up periods according to different thyroid profile parameters

\begin{tabular}{|c|c|c|c|c|c|}
\hline & & Baseline & Follow up 6 months & 1 year & $p$ \\
\hline \multirow[t]{12}{*}{$\mathrm{TSH}^{\#}$} & Total & $n=48$ & $n=48$ & $n=48$ & \\
\hline & Median (min.-max.) & $1.2(0.3-11)$ & $1.3(0.4-5.2)$ & $1(0.3-5.2)$ & 0.062 \\
\hline & Mean \pm SD & $1.6 \pm 1.7$ & $1.4 \pm 1$ & $1.4 \pm 1.1$ & \\
\hline & Avonex & $n=8$ & $n=8$ & $n=8$ & \\
\hline & Median (min.-max.) & $1.7(0.3-11)$ & $0.8(0.5-5.2)$ & $0.8(0.5-5.2)$ & 0.446 \\
\hline & Mean \pm SD & $2.9 \pm 3.5$ & $1.8 \pm 1.8$ & $1.7 \pm 1.8$ & \\
\hline & Betaferon & $n=19$ & $n=19$ & $n=19$ & \\
\hline & Median (min.-max.) & $0.8(0.3-2.9)$ & $0.8(0.4-3)$ & $0.7(0.3-3)$ & 0.692 \\
\hline & Mean \pm SD & $1.1 \pm 0.7$ & $1.1 \pm 0.8$ & $1.1 \pm 0.8$ & \\
\hline & Rebeif & $n=21$ & $n=21$ & $n=21$ & \\
\hline & Median (min.-max.) & $1.4(0.4-3.5)$ & $1.6(0.4-3.3)$ & $1.8(0.3-3)$ & $0.028^{*}$ \\
\hline & Mean \pm SD & $1.5 \pm 0.8$ & $1.6 \pm 0.7$ & $1.6 \pm 0.9$ & \\
\hline \multirow[t]{12}{*}{$\mathrm{FT}^{\$}$} & Total & $n=48$ & $n=48$ & $n=48$ & \\
\hline & Median (min.-max.) & $3.1(1.2-3.8)$ & $3.2(1.8-3.9)$ & $3.3(1.8-3.9)$ & $0.007^{*}$ \\
\hline & Mean \pm SD & $3.0 \pm 0.5$ & $3.1 \pm 0.4$ & $3.2 \pm 0.4$ & \\
\hline & Avonex & $n=8$ & $n=8$ & $n=8$ & \\
\hline & Median (min.-ax.) & $3.1(1.2-3.8)$ & $2.9(2.4-3.7)$ & $3.1(2.4-3.7)$ & 0.408 \\
\hline & Mean \pm SD & $2.8 \pm 0.8$ & $2.9 \pm 0.4$ & $3.1 \pm 0.4$ & \\
\hline & Betaferon & $n=19$ & $n=19$ & $n=19$ & \\
\hline & Median (min.-max.) & $3.2(2-3.7)$ & $3.3(1.8-3.9)$ & $3.3(1.8-3.9)$ & 0.101 \\
\hline & Mean \pm SD & $3.1 \pm 0.4$ & $3.2 \pm 0.5$ & $3.2 \pm 0.5$ & \\
\hline & Rebeif & $n=21$ & $n=21$ & $n=21$ & \\
\hline & Median (min.-max.) & $3(2-3.5)$ & $3.2(2.2-3.6)$ & $3.3(2.2-3.6)$ & $0.028^{*}$ \\
\hline & Mean \pm SD & $3 \pm 0.4$ & $3.2 \pm 0.3$ & $3.2 \pm 0.4$ & \\
\hline \multirow[t]{12}{*}{$\mathrm{FT}^{\text {s }}$} & Total & $n=48$ & $n=48$ & $n=48$ & \\
\hline & Median (min.-max.) & $1.2(0.3-1.9)$ & $1.2(0.8-1.9)$ & $1.2(0.8-1.9)$ & 0.303 \\
\hline & Mean \pm SD & $1.2 \pm 0.3$ & $1.2 \pm 0.3$ & $1.2 \pm 0.3$ & \\
\hline & Avonex & $n=8$ & $n=8$ & $n=8$ & \\
\hline & Median (min.-max.) & $1.2(0.3-1.5)$ & $1.3(0.8-1.9)$ & $1.3(0.8-1.9)$ & 0.398 \\
\hline & Mean \pm SD & $1.1 \pm 0.4$ & $1.3 \pm 0.3$ & $1.3 \pm 0.3$ & \\
\hline & Betaferon & $n=19$ & $n=19$ & $n=19$ & \\
\hline & Median (min.-max.) & $1.2(0.7-1.8)$ & $1.1(0.9-1.8)$ & $1.1(0.9-1.9)$ & 0.615 \\
\hline & Mean \pm SD & $1.2 \pm 0.3$ & $1.2 \pm 0.3$ & $1.2 \pm 0.3$ & \\
\hline & Rebeif & $n=21$ & $n=21$ & $n=21$ & \\
\hline & Median (min.-max.) & $1.2(0.8-1.9)$ & $1.2(0.8-1.9)$ & $1.2(0.8-1.9)$ & 0.826 \\
\hline & Mean \pm SD & $1.2 \pm 0.3$ & $1.2 \pm 0.2$ & $1.2 \pm 0.2$ & \\
\hline
\end{tabular}

$p, p$ value for comparing between the different periods

*Statistically significant at $p \leq 0.05$

\# $p$ value for Friedman test

${ }^{\$} p$ value for $F$ test (ANOVA) with repeated measures

the initial interview ending at December 2018. The patients were drug-naive patients of both sex diagnosed with multiple sclerosis according to Macdonald's criteria 2010.
We compared thyroid profile in the selected patients according to the specific interferon beta given and followed our patients over 12 months. We found an overall statistically significant increase in free T3 levels 


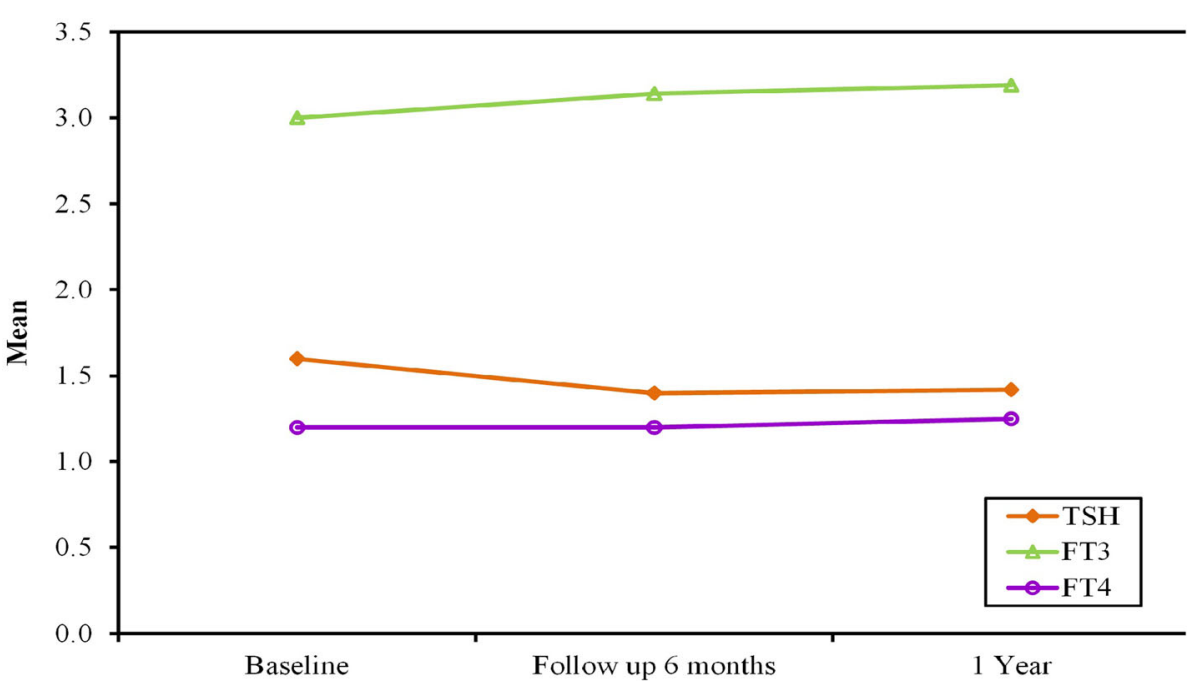

Fig. 2 Comparison between the whole group at baseline, 6-, and 12-month follow-up periods according to different thyroid profile parameters

in the whole studied group and specifically in those treated with Rebif and Betaferon. It also showed a trend to increase in those treated with Avonex but without statistical significance.

TSH level showed a tendency to decrease with followup in the whole group and in the three subgroups but it showed statistical significance only in those treated with Rebif.

This contradicts with some previous studies that did not prove any significant change in thyroid profile in the studied cases. Most of them showed significant change in thyroid antibodies profile with no change in routine thyroid profile.

A recent study in 2018 was done in Ain Shams University in about 100 drug-naïve MS patients compared with age-matched healthy volunteers; it showed normal thyroid profile including TSH, FT3, and FT4 in both groups [8].

Seyfert and colleagues found in a prospective study on 100 drug-naïve multiple sclerosis patients and 97 healthy control no significant change in thyroid profile in both groups although there was a significant change in thyroid antibodies [11].

Another study done by Petek-Balci reported an association between drug-naïve MS patients and thyroid autoimmunity among 106 MS patients by comparing them with healthy volunteers where there was a significant difference between both groups regarding thyroid antibodies with no significant difference regarding thyroid profile between both groups as was found in this study [12].

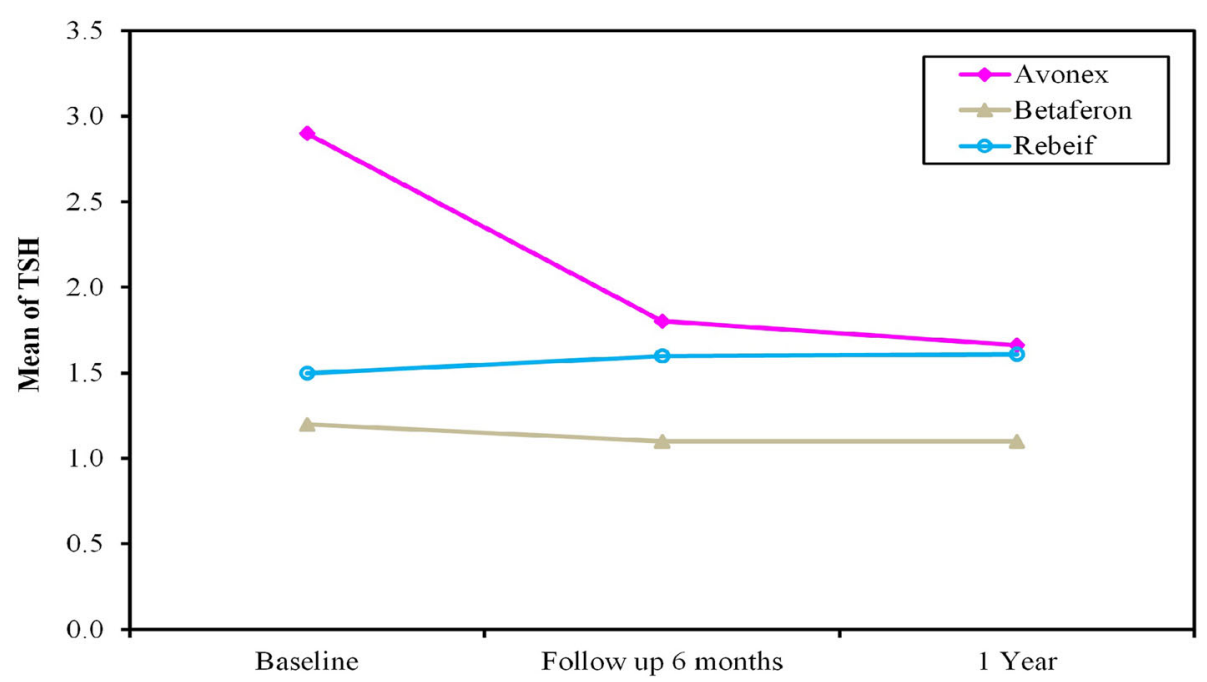

Fig. 3 Comparison between each separate drug groups at baseline, 6-, and 12-month follow-up periods according to TSH 


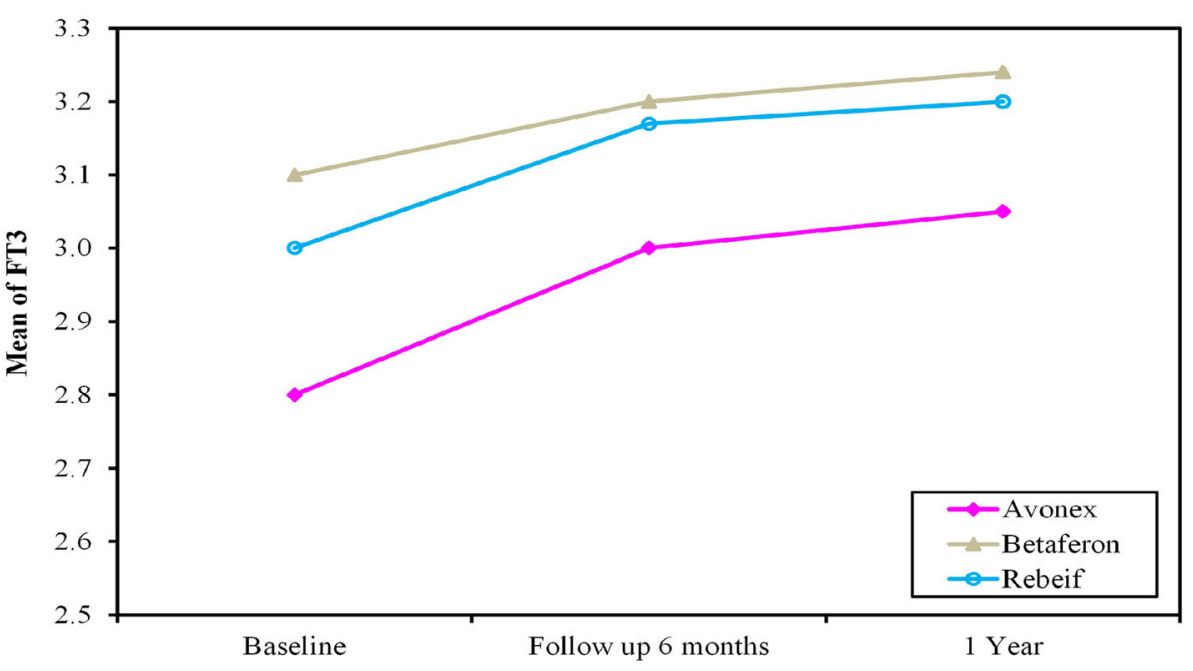

Fig. 4 Comparison between each separate drug groups at baseline, 6-, and 12-month follow-up periods according to FT3

These mentioned studies adopted a comparative structure that compared healthy volunteers with MS patients at a 6-month follow-up and this may partially explain the contradiction with our work that pointed a longer follow-up interval of thyroid profile up to 12 months in the patients without comparison with other cohorts, as most of the significant changes in the profile were noted at 12-month follow-up visits, while the results at 6 months follow-up in our study were comparative to the results of the mentioned studies.

This may be a point of further research with a longer follow-up interval to check the net effect of interferon therapy on the thyroid profile at longer time frames and in different MS patient's subgroups.

\section{Conclusions}

The study showed a statistically significant increase in serum-free T3 levels in those treated with interferon beta therapy in a follow-up of 1 year. It showed also a decrease in serum-free T4 levels without statistical significance. TSH levels showed a tendency towards increase without statistical significance.

Our study adopted a longer follow-up for 12 months in comparison to previous works and this helped us to detect the subclinical changes in thyroid profile in the selected cases in a longer time span. This can be a recommendation to adopt a regular screening of thyroid functions in patients receiving beta interferon therapy.

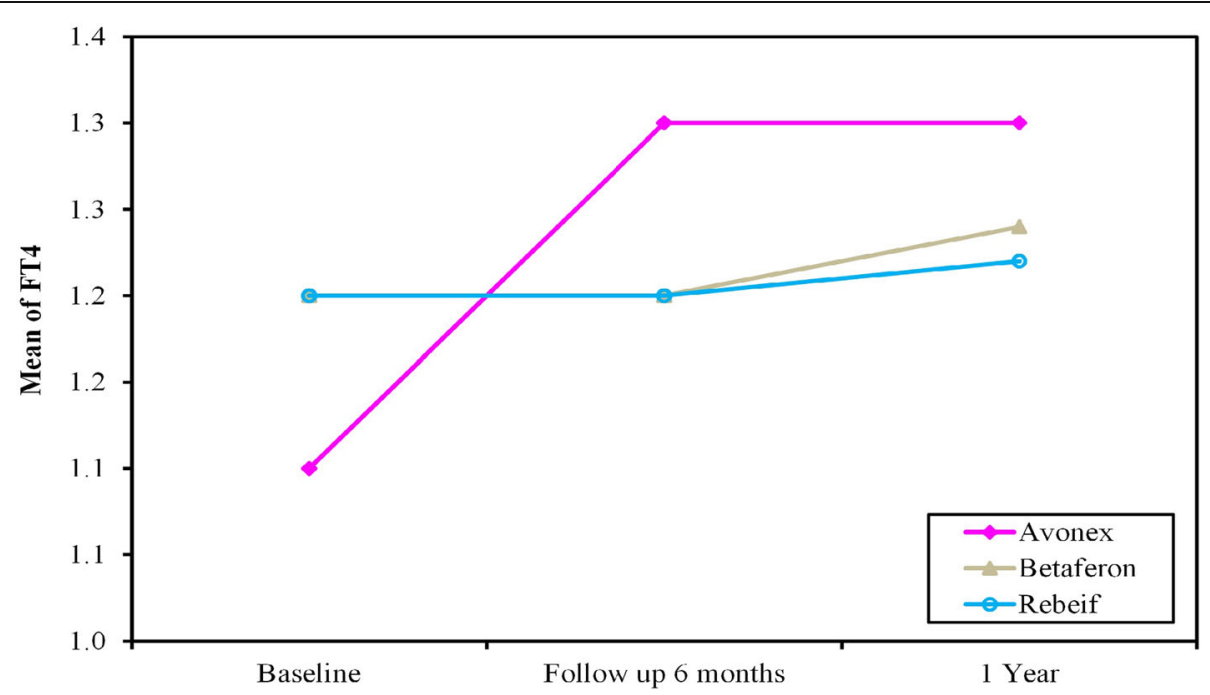

Fig. 5 Comparison between each separate drug groups at baseline, 6-, and 12-month follow-up periods according to FT4 
Our study was limited by our protocol to assess thyroid antibodies only in those with increase in TSH levels, so it is highly recommended to do a wider scale study on a larger sample including all the thyroid antibodies. Comparison between each separate drug groups at baseline, 6-, and 12-month follow-up periods in thyroid profile assessment is recommended to find correlation between thyroid antibody levels and routine thyroid hormone profile in the different groups of patients on interferon beta therapy-if any-and compare this with the given results in our current study.

\section{Abbreviations}

MS: Multiple sclerosis; TSH: Thyroid stimulating hormone; FT3: Free tri-iodo thyronine; FT4: Free thyroxine; AITDs: Autoimmune thyroid diseases; IFN$\beta$ : Interferon beta

\section{Acknowledgements}

We are deeply owing to the help of our eminent Alexandria MS clinic team: Amira Sayed, assistant lecturer of neurology and Shehab Ashraf, senior resident for their great efforts in interviewing and collecting data from our patients. Without their help, we would have met lots of difficulties in this work.

\section{Authors' contributions}

FT chose the idea and put the proposal. HD is the corresponding author, researched literature and conceived the study and was involved in protocol development, gaining ethical approval, and data analysis. HD wrote the first draft of the manuscript. All authors reviewed and edited the manuscript and approved the final version of the manuscript.

\section{Funding}

No sources of funding.

\section{Availability of data and materials}

Data are available in the manuscript along with any conditions for access.

\section{Ethics approval and consent to participate}

Ethical approval was obtained from the Ethics Committee (EC) of the Alexandria Faculty of Medicine which is constituted and operates according to the International Conference on Harmonisation-Good Clinical Practice ICH GCP guidelines (Food and Drug Administration guideline) and applicable local and institutional regulations and guidelines which govern EC operation. The approval was obtained by the monthly meeting of EC on 11th February 2018. There is no registry number for the ethics committee acceptance. Informed written consents were taken from the patients before participation in the study.

\section{Consent for publication}

Not applicable

\section{Competing interests}

The authors declare that they have no competing interests.

Received: 12 May 2020 Accepted: 18 January 2021

Published online: 01 February 2021

\section{References}

1. Durelli L, Oggero A, Verdun E, Isoardo GL, Barbero P, Bergamasco B, et al. Throid function and anti-thyroid antibodies in MS patients screened for interferon treatment. A multicenter study. J Neurol Sci. 2001;193(1):17-22.

2. Marrie RA, Yu BN, Leung S, Elliott L, Warren S, Wolfson C, et al. The incidence and prevalence of fibromyalgia are higher in multiple sclerosis than the general population: a population-based study. Mult Scler Relat Disord. 2012;1 (4):162-7.

3. Niederwieser G, Buchinger W, Bonelli RM, Berghold A, Reisecker F, Költringer $P$, et al. Prevalence of autoimmune thyroiditis and non-immune thyroid disease in multiple sclerosis. J Neurol. 2003;250(6):672-5.
4. Chen C, Wu N, Watson C. Multiple sclerosis patients who are stable on interferon therapy show better outcomes when staying on same therapy than patients who switch to another interferon. Clinicoecon Outcomes Res. 2018;10:723-30.

5. Hamdy SM, Abdel-naseer M, Shalaby NM, et al. Characteristics and predictors of progression in an Egyptian multiple sclerosis cohort: a multicenter registry study. Neuropsychiatr Dis Treat. 2017;13:1895-903. https://doi.org/10.2147/NDT.

6. Shehata HS, ELmazny A, Hamdy SM, et al. Interferon-beta-induced headache in patients with multiple sclerosis: frequency and characterization. Journal of Pain Research. 2020;13:537-45.

7. Sloka JS, Phillips PW, Stefanelli M, Joyce C. Co-occurrence of autoimmunenthyroid disease in a multiple sclerosis cohort. J Autoimmune Dis. 2005;2:9.

8. Mohammed MS, Shoeib NH, Sabry IM, Abd El Gawad DM, Bahaaeldin AM, Adly NN. Evaluation of thyroid functions in patients with multiple sclerosis before and after treatment with interferon beta. Thyroid Disorders Ther. 2018;7:1.

9. Polman $\mathrm{CH}$, Reingold SC, Banwell B, et al. Diagnostic criteria for multiple sclerosis: 2010 revisions to the McDonald criteria. Ann Neurology. 2011; 69(2):292-302.

10. Cao H, Peyrodie L, Boudet S, et al. Expanded Disability Status Scale (EDSS) estimation in multiple sclerosis from posturographic data. Gait Posture. 2013; 37(2):242-5. https://doi.org/10.1016/.gaitpost.2012.07.011.

11. Seyfert S, Klapps P, Meisel C. Multiple sclerosis and other immunologic diseases. Acta Neurol Scand. 1990;81:37-42.

12. Petek-Balci B, Yayla V, Ozer F. Multiple sclerosis and Hashimoto thyroiditis: two cases. Neurologist. 2005;11(5):301-4.

\section{Publisher's Note}

Springer Nature remains neutral with regard to jurisdictional claims in published maps and institutional affiliations.

\section{Submit your manuscript to a SpringerOpen ${ }^{\circ}$ journal and benefit from:}

- Convenient online submission

- Rigorous peer review

- Open access: articles freely available online

High visibility within the field

- Retaining the copyright to your article

Submit your next manuscript at $\boldsymbol{\nabla}$ springeropen.com 